\title{
The further generalization on the inequalities for Hadamard products of any number of invertible Hermitian matrices
}

\author{
Meixiang Chen ${ }^{1,2}$, Qinghua Chen ${ }^{2}$, Zhongpeng Yang ${ }^{1,3^{*}}$, Xiaoxia Feng $^{3}$ and Zhixing Lin ${ }^{1}$
}

\author{
"Correspondence: \\ yangzhongpeng@126.com \\ 'School of Mathematics, Putian \\ University, Putian, 351100, P.R. China \\ ${ }^{3}$ School of Mathematics and \\ Statistics, Minnan Normal University, \\ Zhangzhou, 363000, P.R. China \\ Full list of author information is \\ available at the end of the article
}

\begin{abstract}
Without 'positive definiteness' demanded in the present papers, the forward and reverse inequalities for Hadamard products of any number of invertible Hermitian matrices are obtained, and the sufficient and necessary conditions for the equations in these inequalities are given. As Hermitian positive matrices naturally satisfy the added constraints, these results generalize and improve the corresponding results in the present papers. Beyond that, with no demand of 'positive definiteness', these forward and backward inequalities are not determined mutually any longer.
\end{abstract}

Keywords: Hermitian matrix; Hadamard product; matrix inequality; equation condition

\section{Introduction}

Throughout the paper, we assume $C^{m \times n}$ is the set of $m \times n$ complex matrices, $I$ is a identity matrix, $E_{i i}$ is a diagonal matrix with 1 at its $(i, i)$ th position and 0 elsewhere, and $Z_{n}=$ $\left[E_{11}, E_{22}, \ldots, E_{n n}\right]^{*} \in C^{n^{2} \times n}$ is a selection matrix. $A^{*}$ stands for the conjugate transpose of $A \in C^{m \times n}$. The matrix $A$ is Hermitian if $A^{*}=A$, denoted by $A \in H(n)$. Furthermore, we denote by $H_{0}^{+}(n)$ and $H^{+}(n)$ the sets of Hermitian semi-positive matrices and Hermitian positive matrices, respectively. Recall that $A, B$ are said to have the inequality $A \geq B$ or $B \leq A$, if $A-B \in H_{0}^{+}(n)$. In particular, $A \in H_{0}^{+}(n)$ (resp. $A \in H^{+}(n)$ ), denoted by $A \geq 0$ (resp. $A>0$ ), and denoted by $A^{\frac{1}{2}}$ the square root of $A$. For a positive integer $k$, we have $\alpha(k) \subseteq$ $\left\langle n^{k}\right\rangle=\left\{1,2, \ldots, n^{k}\right\}, \alpha^{\prime}(k)=\left\langle n^{k}\right\rangle-\alpha(k)$. Especially, $\alpha=\alpha(1) \in\langle n\rangle$. For $A \in C^{n \times n}, A(\alpha, \beta)$ denotes the submatrix of $A$ lying in rows indexed by $\alpha$ and the columns indexed by $\beta$ and $A(\alpha, \alpha)=A(\alpha)$. The Hadamard and Kronecker products of $A=\left(a_{i j}\right), B=\left(B_{i j}\right)$ are defined as $A \circ B=\left(a_{i j} b_{i j}\right)$ and $A \otimes B=\left(a_{i j} B\right)$, respectively. If $A X A=X, X A X=X,(A X)^{*}=A X$, and $(X A)^{*}=X A$, then $X$ is said to be a Moore-Penrose generalized inverse of $A$, denoted by $X=A^{+}$.

Recall that if every diagonal element of $R \in H_{0}^{+}(n)$ is 1 , then $R$ is said to be a correlation matrix, in symbol $R \in \mathrm{CH}_{0}^{+}(n)$ (see [1]). The invertible matrix $A \in H_{0}^{+}(n)$ implies $A \in H^{+}(n)$, thus the set of invertible matrices $\mathrm{CH}^{+}(n) \subset \mathrm{H}^{+}(n)$.

By multivariate analysis, Styan obtained the inequalities as follows in 1973 (see [1, Theorem 4.1, Corollary 4.2(4.21) and Corollary 4.3]):

$$
R \circ R-2\left(R^{-1} \circ R+I\right)^{-1} \geq 0, \quad R \in C H^{+}(n)
$$

O2014 Chen et al.; licensee Springer. This is an Open Access article distributed under the terms of the Creative Commons Attribution License (http://creativecommons.org/licenses/by/2.0), which permits unrestricted use, distribution, and reproduction in any medium, provided the original work is properly cited. 


$$
\begin{aligned}
& R^{-1} \circ R+I-2(R \circ R)^{-1} \geq 0, \quad R \in C H^{+}(n) ; \\
& A \circ A-2(A \circ I)\left(A^{-1} \circ A+I\right)^{-1}(A \circ I) \geq 0, \quad A \in H^{+}(n) ; \\
& A^{-1} \circ A+I-2(A \circ I)(A \circ A)^{-1}(A \circ I) \geq 0, \quad A \in H^{+}(n) .
\end{aligned}
$$

Meanwhile, Styan pointed out 'A matrix-theoretic proof of Theorem 4.1 would be of interest.' (see [1]).

Many papers [2-10] focus on the generalization of inequalities (1.1)-(1.4) to Hermitian (semi-)positive matrices by matrix methods. As a generalization of the usual Hadamard product, the Khatri-Rao product $[5,6,8]$ has many similar properties to the Hadamard product (see [5]), thus here we only focus on the Hadamard product, a basic product. Referring to [2] and [6], we denote

$$
\prod_{l=1}^{k} \circ A_{l}=A_{1} \circ A_{2} \circ \cdots \circ A_{k}, \quad \prod_{l=1}^{k} \otimes A_{l}=A_{1} \otimes A_{2} \otimes \cdots \otimes A_{k}, \quad A_{l} \in C^{n \times n}, l=1,2, \ldots, k .
$$

In 1979, Ando [2] obtained the following.

Proposition 1.1 (see [2, Theorem 20]) Let $A \in H^{+}(n)$, for any positive integer $k(\geq 2)$, one has

$$
\prod_{l=1}^{k} \circ A \geq k(A \circ I)^{k-1}\left[A^{-1} \circ\left(\prod_{l=1}^{k-1} \circ A\right)+(k-1)(A \circ I)^{k-2}\right]^{-1}(A \circ I)^{k-1} .
$$

When $k=2$, the inequality (1.5) implies (1.3). Ando also made clear, by applying the method of Proposition 1.1, that one has the following.

Proposition 1.2 (see [2, p.239]) Let $A, B \in H^{+}(n)$. Then

$$
A \circ B \geq(A \circ I+B \circ I)\left(A \circ B^{-1}+A^{-1} \circ B+2 I\right)^{-1}(A \circ I+B \circ I) .
$$

In 2000, Zhang showed the following.

Proposition 1.3 (see [3, Application 4]) Let $A, B \in H^{+}(n)$. Then

$$
A \circ B^{-1}+A^{-1} \circ B+2 I \geq(A \circ I+B \circ I)(A \circ B)^{-1}(A \circ I+B \circ I) .
$$

As applications, (1.2) and (1.4) were also given by Visick in 2000 (see [4, Theorem 20]). Moreover, Al Zhour and Kilicman obtained a matrix inequality as follows in 2006 (see [6, Theorem 4.4]):

$$
\begin{gathered}
\left(A_{1} \circ \prod_{l=2}^{k} \circ A_{l}^{\frac{1}{2}} A_{l}^{+\frac{1}{2}}+A_{1}^{\frac{1}{2}} A_{1}^{+\frac{1}{2}} \circ \prod_{l=2}^{k} \circ A_{l}\right)\left(\prod_{l=1}^{k} \circ A_{l}\right)^{+} \\
\quad \times\left(A_{1} \circ \prod_{l=2}^{k} \circ A_{l}^{\frac{1}{2}} A_{l}^{+\frac{1}{2}}+A_{1}^{\frac{1}{2}} A_{1}^{+\frac{1}{2}} \circ \prod_{l=2}^{k} \circ A_{l}\right)
\end{gathered}
$$




$$
\begin{aligned}
& \leq 2\left(\prod_{l=1}^{k} \circ A_{l}^{\frac{1}{2}} A_{l}^{+\frac{1}{2}}\right)+\left(A_{1} \circ \prod_{l=2}^{k} \circ A_{l}^{+}\right)+\left(A_{1}^{+} \circ \prod_{l=2}^{k} \circ A_{l}\right), \\
& A_{l} \in H_{0}^{+}(n), l=1,2, \ldots, k ; \\
&\left(A \circ B^{\frac{1}{2}} B^{+\frac{1}{2}}+A^{\frac{1}{2}} A^{+\frac{1}{2}} \circ B\right)(A \circ B)^{+}\left(A \circ B^{\frac{1}{2}} B^{+\frac{1}{2}}+A^{\frac{1}{2}} A^{+\frac{1}{2}} \circ B\right) \\
& \leq A \circ B^{+}+A^{+} \circ B+2 A^{\frac{1}{2}} A^{+\frac{1}{2}} \circ B^{\frac{1}{2}} B^{+\frac{1}{2}}, \quad A, B \in H_{0}^{+}(n) .
\end{aligned}
$$

For $A \in H_{0}^{+}(n)$, by [11], one has $A^{\frac{1}{2}} A^{+\frac{1}{2}}=A A^{+}$. Because of the commutativity of the Hadamard product, the inequality (1.9) is equivalent to [7, Proposition 1], [8, Corollary 1(5)], it could be viewed as a generalization of (1.4) over Hermitian semi-positive matrices.

For $A, B \in H^{+}(n)$, (1.6) and (1.7) are the forward and backward inequalities to each other, and determined mutually as well (Theorem 2.6), [8] has ever called them as companion inequalities. Of course, (1.1) and (1.2), (1.3) and (1.4) are also the companion inequalities determined by each other (Lemma 2.4). In fact, [9, Theorem 1] shows us the backward inequality which is companied to (1.9).

Papers [1-10] all discuss on Hermitian (semi-)positive matrices. However, the following Example 1.4 illustrates that the condition 'positive definiteness' is not necessary for the inequality (1.7) to hold.

Example 1.4 Let $A=\frac{1}{2}\left[\begin{array}{cc}-1 & 1 \\ 1 & 1\end{array}\right], B=\frac{1}{3}\left[\begin{array}{cc}1 & 1 \\ 1 & -2\end{array}\right] \in H(2)$. Then the matrix inequality (1.7) holds, this is because

$$
\left(A \circ B^{-1}+A^{-1} \circ B+2 I\right)-((A+B) \circ I)(A \circ B)^{-1}((A+B) \circ I)=\left[\begin{array}{ll}
1 & 1 \\
1 & 1
\end{array}\right] \in H_{0}^{+}(2) .
$$

Example 1.5 Let $A=\left[\begin{array}{cc}-1 & 1 \\ 1 & 1\end{array}\right], B=\left[\begin{array}{cc}-1 & 1 \\ 1 & 2\end{array}\right] \in H(2)$. Then the inequality (1.7) does not hold anymore, this is because

$$
\left(A \circ B^{-1}+A^{-1} \circ B+2 I\right)-((A+B) \circ I)(A \circ B)^{-1}((A+B) \circ I)=\frac{1}{6}\left[\begin{array}{ll}
-29 & -31 \\
-31 & -34
\end{array}\right] \text {. }
$$

Example 1.5 indicates that, in general, the matrix inequality (1.7) does not hold for all invertible Hermitian matrices. Hence we will add some constraint conditions in our discussion.

In this paper, without the 'positive definiteness' demanded in the inequality (1.5), we will show inequalities and their companion forms for any number of invertible Hermitian matrices and get sufficient and necessary conditions for the equations in the related inequalities to hold. In view of $[12,13]$ etc., we see the discussion of the equation conditions for the inequalities is significant. Further, Hermitian positive matrices satisfy the added constraints naturally, thus these results generalize and improve the corresponding results in the present literature. However, with no demand of 'positive definiteness', the new forward and backward companion inequalities are not determined mutually. 


\section{Preliminaries}

For matrices of appropriate sizes, in view of [14, Proposition 4.2.14] and [15, Propositions 7.13-7.17], by induction to $k$, it follows that

$$
\begin{aligned}
& (A \otimes B) \otimes C=A \otimes(B \otimes C) ; \\
& (A+B) \otimes C=A \otimes C+B \otimes C, \quad C \otimes(A+B)=C \otimes A+C \otimes B ; \\
& \left(\prod_{l=1}^{k} \otimes A_{l}\right)^{*}=\prod_{l=1}^{k} \otimes A_{l}^{*}, \quad A_{l} \in C^{m \times n}, l=1,2, \ldots, k ; \\
& \left(\prod_{l=1}^{k} \otimes A_{l}\right)\left(\prod_{l=1}^{k} \otimes B_{l}\right)=\prod_{l=1}^{k} \otimes A_{l} B_{l}, \quad \prod_{l=1}^{k}\left(A_{l} \otimes B_{l}\right)=\left(\prod_{l=1}^{k} A_{l}\right) \otimes\left(\prod_{l=1}^{k} B_{l}\right) ; \\
& \left(\prod_{l=1}^{k} \otimes A_{l}\right)^{-1}=\prod_{l=1}^{k} \otimes A_{l}^{-1}, \quad \text { any of } A_{l} \in C^{n \times n} \text { is invertible, } l=1,2, \ldots, k ; \\
& A_{l} \in H(n)\left(H_{0}^{+}(n), H^{+}(n)\right), \quad l=1,2, \ldots, k \\
& \Rightarrow \prod_{l=1}^{k} \otimes A_{l} \in H\left(n^{k}\right)\left(H_{0}^{+}\left(n^{k}\right), H^{+}\left(n^{k}\right)\right) .
\end{aligned}
$$

Lemma 2.1 Let $A_{l} \in C^{n \times n}, l=1,2, \ldots, k$. Then

$$
\begin{aligned}
& \prod_{l=1}^{k} \circ A_{l}=\left(\prod_{l=1}^{k} \otimes A_{l}\right)(\alpha(k)) \in C^{n \times n}, \quad \alpha(k)=\left(\alpha_{k}^{(1)}, \alpha_{k}^{(2)}, \ldots, \alpha_{k}^{(n)}\right) \subset\left\langle n^{k}\right\rangle ; \\
& \alpha_{k}^{(l)}=\left[(l-1)\left(n^{k}-1\right) /(n-1)\right]+1, \quad 1 \leq l \leq n .
\end{aligned}
$$

Proof In view of [4, Theorem 1 and Corollary 2] or [15, Proposition 7.3.1] yields

$$
A_{1} \circ A_{2}=P(n, 2)^{*}\left(A_{1} \otimes A_{2}\right) P(n, 2), \quad P(n, 2)=Z_{n} \in C^{n^{2} \times n}, A_{1}, A_{2} \in C^{n \times n},
$$

that is,

$$
A_{1} \circ A_{2}=\left(A_{1} \otimes A_{2}\right)(\alpha(2)), \quad \alpha(2)=\left(1, n+2,2 n+3, \ldots, n^{2}\right) \subset\left\langle n^{2}\right\rangle,
$$

which indicates (2.7) and (2.8) hold for $k=2$.

By (2.9), (2.1), (2.3), and (2.4), similar to [10, Lemma 2.1], it follows that

$$
\begin{aligned}
A_{1} \circ A_{2} \circ A_{3} & =A_{1} \circ\left(A_{2} \circ A_{3}\right) \\
& =P(n, 2)^{*}\left[I A_{1} I \otimes P(n, 2)^{*}\left(A_{2} \otimes A_{3}\right) P(n, 2)\right] P(n, 2) \\
& =P(n, 2)^{*}(I \otimes P(n, 2))^{*}\left(A_{1} \otimes A_{2} \otimes A_{3}\right)(I \otimes P(n, 2)) P(n, 2),
\end{aligned}
$$

that is,

$$
A_{1} \circ A_{2} \circ A_{3}=P(n, 3)^{*}\left(A_{1} \otimes A_{2} \otimes A_{3}\right) P(n, 3) \in C^{n \times n}, \quad P(n, 3)=(I \otimes P(n, 2)) Z_{n} . \quad(2.10)
$$


As $E_{i i} E_{j j}=E_{i i}(i=j), E_{i i} E_{j j}=0(i \neq j)$, and $E_{i i} \in H(n)$, by (2.9) and (2.10), we see

$$
\begin{aligned}
P(n, 3) & =\operatorname{diag}(P(n, 2), P(n, 2), \ldots, P(n, 2)) Z_{n} \\
& =\left(E_{11}, 0, \ldots, 0 ; 0, E_{22}, 0, \ldots, 0 ; \ldots ; 0, \ldots, 0, E_{n n}\right) \in C^{n^{3} \times n}
\end{aligned}
$$

which shows the $\alpha(3)$ determined by $P(n, 3)$ satisfies (2.8), then (2.7) holds.

Just as the proof of [10, Lemma 2.1], similarly, one has

$$
\prod_{l=1}^{k} \circ A_{l}=P(n, k)^{*}\left(\prod_{l=1}^{k} \otimes A_{l}\right) P(n, k), \quad P(n, k)=(I \otimes P(n, k-1)) Z_{n} \in C^{n^{k} \times n},
$$

thus the $\alpha(k)$ determined by $P(n, k)$ satisfies (2.8), then (2.7) follows by (2.11).

The proof method of Lemma 2.1 plays a great role in discussing the matrix inequalities for Khatri-Rao products of any finite number of positive matrices (see [16, Lemma 2.1], [6, Lemmas 2.1 and 2.2]). Recently, [17, Theorem 3] has also discussed a similar problem to Lemma 2.1, but our results (2.7) and (2.8) should be more convenient in applications.

When $A \in C^{n \times n}$, if $\alpha=\alpha(1) \in\langle n\rangle$ and $A(\alpha)$ is invertible, we call

$$
A / \alpha=A\left(\alpha^{\prime}\right)-A\left(\alpha^{\prime}, \alpha\right) A(\alpha)^{-1} A\left(\alpha, \alpha^{\prime}\right), \quad \alpha^{\prime}=\langle n\rangle-\alpha, A(\alpha)^{-1}=(A(\alpha))^{-1}
$$

the Schur complement of $A(\alpha)$ in $A$ (see $[8,18,19])$.

Lemma 2.2 Let $A \in H(n)$ and $A\left(\alpha^{\prime}\right)$ be both invertible. Then both of $A / \alpha^{\prime}$ and $A^{-1}(\alpha)$ are invertible as well, and $\left(A / \alpha^{\prime}\right)^{-1}=A^{-1}(\alpha)$.

Proof By assumption, there exists a permutation matrix $U$ such that

$$
U^{*} A U=\left[\begin{array}{cc}
A(\alpha) & A\left(\alpha, \alpha^{\prime}\right) \\
A\left(\alpha^{\prime}, \alpha\right) & A\left(\alpha^{\prime}\right)
\end{array}\right], U^{*} A^{-1} U=\left[\begin{array}{cc}
A^{-1}(\alpha) & A^{-1}\left(\alpha, \alpha^{\prime}\right) \\
A^{-1}\left(\alpha^{\prime}, \alpha\right) & A^{-1}\left(\alpha^{\prime}\right)
\end{array}\right] \in H(n)
$$

Since both of $A$ and $A\left(\alpha^{\prime}\right)$ are invertible, by (2.12), one has a matrix $V=\left[\begin{array}{cc}I & 0 \\ -A\left(\alpha^{\prime}\right)^{-1} A\left(\alpha^{\prime}, \alpha\right) & I\end{array}\right]$ such that

$$
(U V)^{*} A(U V)=\operatorname{diag}\left(A / \alpha^{\prime}, A\left(\alpha^{\prime}\right)\right)=A / \alpha^{\prime} \oplus A\left(\alpha^{\prime}\right) \in H(n),
$$

thus $A / \alpha^{\prime}$ is invertible. By (2.12),

$$
U^{*} A^{-1} U=\left(U^{*} A U\right)^{-1}=V\left(\left(A / \alpha^{\prime}\right)^{-1} \oplus A\left(\alpha^{\prime}\right)^{-1}\right) V^{*}=\left[\begin{array}{cc}
\left(A / \alpha^{\prime}\right)^{-1} & * \\
* & *
\end{array}\right],
$$

by comparing with (2.12), it follows that $A^{-1}(\alpha)$ is invertible and $\left(A / \alpha^{\prime}\right)^{-1}=A^{-1}(\alpha)$.

When $A \in H^{+}(n)$, it is natural that $A\left(\alpha^{\prime}\right)$ is invertible, hence we could obtain [18, formula (4)] again by Lemma 2.2 . 
Lemma 2.3 Let $C \in C^{n \times n}$ and $A \in H(n)$ be invertible, $\alpha \subset\langle n\rangle, \alpha^{\prime}=\langle n\rangle-\alpha$, if $\left.A^{-1}\left(\alpha^{\prime}\right)\right\rangle 0$, then $A(\alpha)$ is invertible and

$$
C(\alpha)^{*} A(\alpha)^{-1} C(\alpha) \leq\left(C^{*} A^{-1} C\right)(\alpha), \quad \text { where } C(\alpha)^{*}=(C(\alpha))^{*} \text {. }
$$

Moreover, the equation in (2.13) holds if and only if $A^{-1}\left(\alpha^{\prime}, \alpha\right) C(\alpha)+A^{-1}\left(\alpha^{\prime}\right) C\left(\alpha^{\prime}, \alpha\right)=0$.

Proof In this case, there is a permutation matrix $U$ such that (2.12) holds and $U^{*} C U=$ $\left[\begin{array}{cc}C(\alpha) & C\left(\alpha, \alpha^{\prime}\right) \\ C\left(\alpha^{\prime}, \alpha\right) & C\left(\alpha^{\prime}\right)\end{array}\right]$, then

$$
U^{*}\left(C^{*} A^{-1} C\right) U=\left[\begin{array}{cc}
\left(C^{*} A^{-1} C\right)(\alpha) & \left(C^{*} A^{-1} C\right)\left(\alpha, \alpha^{\prime}\right) \\
\left(C^{*} A^{-1} C\right)\left(\alpha^{\prime}, \alpha\right) & \left(C^{*} A^{-1} C\right)\left(\alpha^{\prime}\right)
\end{array}\right] \in H(n) .
$$

By assumption, both of $A^{-1} \in H(n)$ and $A^{-1}\left(\alpha^{\prime}\right)$ are Hermitian and invertible, then by applying Lemma 2.2, $A(\alpha)$ is invertible and

$$
A^{-1} / \alpha^{\prime}=A^{-1}(\alpha)-A^{-1}\left(\alpha, \alpha^{\prime}\right) A^{-1}\left(\alpha^{\prime}\right)^{-1} A^{-1}\left(\alpha^{\prime}, \alpha\right)^{-1}=\left(A^{-1}\right)^{-1}(\alpha)^{-1}=A(\alpha)^{-1} ;
$$

combining with (2.12), there exists $W=\left[\begin{array}{cc}I & 0 \\ -A^{-1}\left(\alpha^{\prime}\right)^{-1} A^{-1}\left(\alpha^{\prime}, \alpha\right) I\end{array}\right]$ such that

$$
\begin{aligned}
& W^{*} U^{*} A^{-1} U W=\operatorname{diag}\left(A^{-1} / \alpha^{\prime}, A^{-1}\left(\alpha^{\prime}\right)\right)=A(\alpha)^{-1} \oplus A^{-1}\left(\alpha^{\prime}\right), \\
& W^{-1} U^{*} C U=\left[\begin{array}{cc}
C(\alpha) & C\left(\alpha, \alpha^{\prime}\right) \\
X & Y
\end{array}\right], \quad X=A^{-1}\left(\alpha^{\prime}\right)^{-1} A^{-1}\left(\alpha^{\prime}, \alpha\right) C(\alpha)+C\left(\alpha^{\prime}, \alpha\right) .
\end{aligned}
$$

By (2.14)-(2.16), as $A^{-1}\left(\alpha^{\prime}\right)>0$, then $\left(C^{*} A^{-1} C\right)(\alpha)-C(\alpha)^{*} A(\alpha)^{-1} C(\alpha)=X^{*} A^{-1}\left(\alpha^{\prime}\right) X \geq$ 0 , that is, (2.13) follows. Meanwhile, the equation in (2.13) holds; therefore $X=0$, it is equivalent to

$$
A^{-1}\left(\alpha^{\prime}\right) X=A^{-1}\left(\alpha^{\prime}, \alpha\right) C(\alpha)+A^{-1}\left(\alpha^{\prime}\right) C\left(\alpha^{\prime}, \alpha\right)=0 .
$$

For a Hermitian positive matrix $A$, one has $A^{-1}\left(\alpha^{\prime}\right)>0$; then we get [3, Theorem 1(7)] again by Lemma 2.3 .

Lemma 2.4 Let $F, G \in H^{+}(n)$. Then

$$
F \geq T G^{-1} T^{*} \quad \Leftrightarrow \quad G \geq T^{*} F^{-1} T \text { and } F=T G^{-1} T^{*} \quad \Leftrightarrow \quad G=T^{*} F^{-1} T .
$$

Proof In this case, $M=\left[\begin{array}{cc}F & T \\ T^{*} & G\end{array}\right] \in H(2 n)$, and there exist invertible matrices $P=\left[\begin{array}{cc}I & 0 \\ -G^{-1} T^{*} & { }_{I}\end{array}\right]$ and $Q=\left[\begin{array}{cc}I-F^{-1} T \\ 0 & I\end{array}\right]$ such that

$$
P^{*} M P=\left(F-T G^{-1} T^{*}\right) \oplus G, \quad Q^{*} M Q=F \oplus\left(G-T^{*} F^{-1} T\right),
$$

which indicates

$$
\begin{aligned}
F \geq T G^{-1} T^{*} & \Leftrightarrow P^{*} M P \in H_{0}^{+}(2 n) \\
& \Leftrightarrow \quad Q^{*} M Q \in H_{0}^{+}(2 n) \quad \Leftrightarrow \quad G \geq T^{*} F^{-1} T,
\end{aligned}
$$




$$
\begin{aligned}
F=T G^{-1} T^{*} & \Leftrightarrow \operatorname{rank} P^{*} M P=\operatorname{rank} G=n \\
& \Leftrightarrow \quad \operatorname{rank} Q^{*} M Q=\operatorname{rank} F=n \quad \Leftrightarrow \quad G=T^{*} F^{-1} T .
\end{aligned}
$$

Lemmas 2.2-2.4 will play an important role in the discussion.

Theorem 2.5 Let $A \in H^{+}(n)$, for any positive integer $k(\geq 2)$. Then

$$
A^{-1} \circ\left(\prod_{l=1}^{k-1} \circ A\right)+(k-1)(A \circ I)^{k-2} \geq k(A \circ I)^{k-1}\left(\prod_{l=1}^{k} \circ A\right)^{-1}(A \circ I)^{k-1} \text {. }
$$

Moreover, the equation in (1.5) holds if and only if the one in (2.17) holds.

Proof As $A \in H^{+}(n)$, by (2.6), $\prod_{l=1}^{k} \circ A, A^{-1} \circ\left(\prod_{l=1}^{k-1} \circ A\right)+(k-1)(A \circ I)^{k-2} \in H^{+}(n)$. Taking $T^{*}=T=\sqrt{k}(A \circ I)^{k-1} \in H(n)$, in view of Proposition 1.1, yields the inequality (1.5), that is, $\prod_{l=1}^{k} \circ A \geq T^{*}\left(A^{-1} \circ\left(\prod_{l=1}^{k-1} \circ A\right)+(k-1)(A \circ I)^{k-2}\right)^{-1} T$, then by Lemma $2.4, A^{-1} \circ$ $\left(\prod_{l=1}^{k-1} \circ A\right)+(k-1)(A \circ I)^{k-2} \geq T^{*}\left(\prod_{l=1}^{k} \circ A\right)^{-1} T$, which shows (2.17) holds, meanwhile, the equation in (1.5) holds; therefore the one in (2.17) holds.

Theorem 2.5 not only leads to the backward inequality (2.17) of (1.5) (in this case, the inequalities (1.5) and (2.17) are mutually determined), but it also shows us that the inequalities (1.1) and (1.2), (1.3) and (1.4) given by Styan are companied and determined by each other (the case of $k=2$ in Theorem 2.5).

By applying Lemma 2.4, Propositions 1.2 and 1.3, with a similar discussion as Theorem 2.5 , we have the following.

Theorem 2.6 Let $A \in H^{+}(n)$. Then the inequalities (1.6) and (1.7) are companied and determined by each other, and the equation in (1.6) holds; therefore the one in (1.7) holds.

\section{Main results}

For Hermitian matrices $A_{l}(l=1,2, \ldots, k)$, unless otherwise specified, we always assume

$$
C=\sum_{t=1}^{k}\left[\left(\prod_{l=1}^{t-1} \otimes A_{l}\right) \otimes I \otimes\left(\prod_{l=t+1}^{k} \otimes A_{l}\right)\right], \quad N=\sum_{t=1}^{k}\left(\left(\prod_{l \neq t} \circ A_{l}\right) \circ I\right) .
$$

Theorem 3.1 Let $A_{l} \in H(n)$ be invertible, $l=1,2, \ldots, k$, and $\alpha(k)$ be as in (2.7) and (2.8), if $\left(\prod_{l=1}^{k} \otimes A_{l}^{-1}\right)\left(\alpha^{\prime}(k)\right)>0$, then $\prod_{l=1}^{k} \circ A_{l}$ is also invertible and

$$
\sum_{t=1}^{k}\left[A_{t}^{-1} \circ\left(\prod_{l \neq t} \circ A_{l}\right)\right]+2 \sum_{1 \leq t<s \leq k}\left[\left(\prod_{l \neq t, s} \circ A_{l}\right) \circ I\right] \geq N\left(\prod_{l=1}^{k} \circ A_{l}\right)^{-1} N .
$$

Moreover, the equation in (3.1) holds if and only if

$$
\left(\prod_{l=1}^{k} \otimes A_{l}^{-1}\right)\left(\alpha^{\prime}(k), \alpha(k)\right) N+\left(\prod_{l=1}^{k} \otimes A_{l}^{-1}\right)\left(\alpha^{\prime}(k)\right) C\left(\alpha^{\prime}(k), \alpha(k)\right)=0 .
$$


Proof By (2.6), we see $C=\sum_{t=1}^{k}\left[\left(\prod_{l=1}^{t-1} \otimes A_{l}\right) \otimes I \otimes\left(\prod_{l=t+1}^{k} \otimes A_{l}\right)\right] \in H\left(n^{k}\right)$, and by (2.5), $\prod_{l=1}^{k} \otimes A_{l}^{-1}=\left(\prod_{l=1}^{k} \otimes A_{l}\right)^{-1}$ is invertible. From our assumption, $\left(\prod_{l=1}^{k} \otimes A_{l}^{-1}\right)\left(\alpha^{\prime}(k)\right)>0$, then by applying (2.7) and Lemma 2.3, $\prod_{l=1}^{k} \circ A_{l}=\left(\prod_{l=1}^{k} \otimes A_{l}\right)(\alpha(k))$ is invertible. By the commutativity of Hadamard products, combining with (2.1)-(2.6) yields

$$
\begin{aligned}
C^{*}\left(\prod_{l=1}^{k} \otimes A_{l}\right)^{-1} C= & C\left(\prod_{l=1}^{k} \otimes A_{l}^{-1}\right) C \\
= & {\left[\sum_{t=1}^{k}\left(\left(\prod_{l=1}^{t-1} \otimes A_{l}\right) \otimes I \otimes\left(\prod_{l=t+1}^{k} \otimes A_{l}\right)\right)\right]\left(\prod_{l=1}^{k} \otimes A_{l}^{-1}\right) } \\
& \times\left[\sum_{t=1}^{k}\left(\left(\prod_{l=1}^{t-1} \otimes A_{l}\right) \otimes I \otimes\left(\prod_{l=t+1}^{k} \otimes A_{l}\right)\right)\right] \\
= & {\left[\sum_{t=1}^{k}\left(\left(\prod_{l=1}^{t-1} \otimes A_{l} A_{l}^{-1}\right) \otimes I A_{t}^{-1} \otimes\left(\prod_{l=t+1}^{k} \otimes A_{l} A_{l}^{-1}\right)\right)\right] } \\
& \times\left[\sum_{t=1}^{k}\left(\left(\prod_{l=1}^{t-1} \otimes A_{l}\right) \otimes I \otimes\left(\prod_{l=t+1}^{k} \otimes A_{l}\right)\right)\right] \\
= & {\left[\sum_{t=1}^{k}\left(\left(\prod_{l=1}^{t-1} \otimes I\right) \otimes A_{t}^{-1} \otimes\left(\prod_{l=t+1}^{k} \otimes I\right)\right)\right] } \\
& \times\left[\sum_{t=1}^{k}\left(\left(\prod_{l=1}^{t-1} \otimes A_{l}\right) \otimes I \otimes\left(\prod_{l=t+1}^{k} \otimes A_{l}\right)\right)\right] \in H\left(n^{k}\right),
\end{aligned}
$$

that is,

$$
\begin{aligned}
& C^{*}\left(\prod_{l=1}^{k} \otimes A_{l}\right)^{-1} C \\
& =\sum_{t=1}^{k}\left(A_{1} \otimes \cdots \otimes A_{t-1} \otimes A_{t}^{-1} \otimes A_{t+1} \otimes \cdots \otimes A_{k}\right) \\
& \quad+2 \sum_{1 \leq t<s \leq k}\left(A_{1} \otimes \cdots \otimes A_{t-1} \otimes I \otimes A_{t+1} \otimes \cdots \otimes A_{s-1} \otimes I \otimes A_{s+1} \otimes \cdots \otimes A_{k}\right) .
\end{aligned}
$$

By (2.7), (2.13), and (3.3), we have $C(\alpha(k))=\sum_{t=1}^{k}\left[\left(\prod_{l \neq t} \circ A_{l}\right) \circ I\right]=N \in H(n)$, and

$$
\begin{aligned}
& \left(C^{*}\left(\prod_{l=1}^{k} \otimes A_{l}\right)^{-1} C\right)(\alpha(k)) \\
& =\sum_{t=1}^{k}\left(\left(\prod_{l=1}^{t-1} \otimes A_{l}\right) \otimes A_{t}^{-1} \otimes\left(\prod_{l=t+1}^{k} \otimes A_{l}\right)\right)(\alpha(k)) \\
& \quad+2 \sum_{1 \leq t<s \leq k}\left(\left(\prod_{l=1}^{t-1} \otimes A_{l}\right) \otimes I \otimes\left(\prod_{l=t+1}^{s-1} \otimes A_{l}\right) \otimes I \otimes\left(\prod_{l=s+1}^{k} \otimes A_{l}\right)\right)(\alpha(k)) \\
& =\sum_{t=1}^{k}\left(A_{1} \circ \cdots \circ A_{t-1} \circ A_{t}^{-1} \circ A_{t+1} \circ \cdots \circ A_{k}\right)
\end{aligned}
$$




$$
\begin{aligned}
& +2 \sum_{1 \leq t<s \leq k}\left(A_{1} \circ \cdots \circ A_{t-1} \circ I \circ A_{t+1} \circ \cdots \circ A_{s-1} \circ I \circ A_{s+1} \circ \cdots \circ A_{k}\right) \\
= & \sum_{t=1}^{k}\left(A_{t}^{-1} \circ\left(\prod_{l \neq t} \circ A_{l}\right)\right)+2 \sum_{1 \leq t<s \leq k}\left(\left(\prod_{l \neq t, s} \circ A_{l}\right) \circ I\right) \\
\geq & C(\alpha(k))^{*}\left(\prod_{l=1}^{k} \otimes A_{l}\right)(\alpha(k))^{-1} C(\alpha(k)) \\
= & {\left[\sum_{t=1}^{k}\left(\left(\prod_{l \neq t} \circ A_{l}\right) \circ I\right)\right]\left(\prod_{l=1}^{k} \circ A_{l}\right)^{-1}\left[\sum_{t=1}^{k}\left(\left(\prod_{l \neq t} \circ A_{l}\right) \circ I\right)\right] } \\
= & N\left(\prod_{l=1}^{k} \circ A_{l}\right)^{-1} N \in H(n),
\end{aligned}
$$

so (3.1) holds.

From Lemma 2.3, the equation in (3.1) holds; therefore

$$
\begin{aligned}
& \left(\prod_{l=1}^{k} \otimes A_{l}^{-1}\right)\left(\alpha^{\prime}(k), \alpha(k)\right) N+\left(\prod_{l=1}^{k} \otimes A_{l}^{-1}\right)\left(\alpha^{\prime}(k)\right) C\left(\alpha^{\prime}(k), \alpha(k)\right) \\
& \quad=\left(\prod_{l=1}^{k} \otimes A_{l}\right)^{-1}\left(\alpha^{\prime}(k), \alpha(k)\right) C(\alpha(k))+\left(\prod_{l=1}^{k} \otimes A_{l}\right)^{-1}\left(\alpha^{\prime}(k)\right) C\left(\alpha^{\prime}(k), \alpha(k)\right)=0 .
\end{aligned}
$$

From Theorems 2.5 and 2.6, we see the inequalities (1.1) and (1.2), (1.3) and (1.4), and the general ones (1.5) and (2.17), (1.6) and (1.7) are companied and determined by each other, hence one of the companion inequalities could be obtained from the other one immediately. However, the following example indicates that the matrix inequality (3.1) is no longer equivalent to its backward inequality, without 'positive definiteness.'

Example 3.2 Let $A, B$ just as the one in Example 1.4, as $\left(A^{-1} \otimes B^{-1}\right)\left(\alpha^{\prime}(2)\right)=\left[\begin{array}{ll}1 & 1 \\ 1 & 2\end{array}\right]>0$, then (1.7) follows by Theorem 3.1, but the inequality (1.6) does not hold; this is because

$$
(A \circ B)-(A \circ I+B \circ I)\left(A^{-1} \circ B+B^{-1} \circ A+2 I\right)^{-1}(A \circ I+B \circ I)=\frac{1}{30}\left[\begin{array}{cc}
-10 & 10 \\
10 & -16
\end{array}\right] \text {. }
$$

Theorem 3.3 Let $A_{l} \in H(n)(l=1,2, \ldots, k)$ be invertible, and $\alpha(k)$ be as in (2.7) and (2.8), if $C$ is invertible and $\left(C^{-1}\left(\prod_{l=1}^{k} \otimes A_{l}\right) C^{-1}\right)\left(\alpha^{\prime}(k)\right)>0$, then $\sum_{t=1}^{k}\left(A_{t}^{-1} \circ\left(\prod_{l \neq t} \circ A_{l}\right)\right)+$ $2 \sum_{1 \leq t<s \leq k}\left(\left(\prod_{l \neq t, s} \circ A_{l}\right) \circ I\right) \in H(n)$ is invertible as well and

$$
N\left[\sum_{t=1}^{k}\left(A_{t}^{-1} \circ\left(\prod_{l \neq t} \circ A_{l}\right)\right)+2 \sum_{1 \leq t<s \leq k}\left(\left(\prod_{l \neq t, s} \circ A_{l}\right) \circ I\right)\right]^{-1} N \leq \prod_{l=1}^{k} \circ A_{l} .
$$

Moreover, the equation in (3.4) holds if and only if

$$
\begin{aligned}
& \left(C^{-1}\left(\prod_{l=1}^{k} \otimes A_{l}\right) C^{-1}\right)\left(\alpha^{\prime}(k), \alpha(k)\right) N+\left(C^{-1}\left(\prod_{l=1}^{k} \otimes A_{l}\right) C^{-1}\right)\left(\alpha^{\prime}(k)\right) C\left(\alpha^{\prime}(k), \alpha(k)\right) \\
& \quad=0 .
\end{aligned}
$$


Proof By (2.6), $C \in H\left(n^{k}\right)$ is invertible, then $\prod_{l=1}^{k} \otimes A_{l}=C^{*}\left(C^{*}\left(\prod_{l=1}^{k} \otimes A_{l}\right)^{-1} C\right)^{-1} C \in H\left(n^{k}\right)$ is also invertible. As

$$
\left(C^{-1}\left(\prod_{l=1}^{k} \otimes A_{l}\right) C^{-1}\right)\left(\alpha^{\prime}(k)\right)=\left(C^{*}\left(\prod_{l=1}^{k} \otimes A_{l}\right)^{-1} C\right)^{-1}\left(\alpha^{\prime}(k)\right)>0,
$$

in view of (3.3), (2.7), and Lemma 2.3 we find that

$$
\begin{aligned}
& \left(C^{*}\left(\prod_{l=1}^{k} \otimes A_{l}\right)^{-1} C\right)(\alpha(k)) \\
& =\sum_{t=1}^{k}\left(A_{t}^{-1} \circ\left(\prod_{l \neq t} \circ A_{l}\right)\right)+2 \sum_{1 \leq t<s \leq k}\left(\left(\prod_{l \neq t, s} \circ A_{l}\right) \circ I\right) \in H(n)
\end{aligned}
$$

is invertible. Then combining with (2.7), (2.13), and Lemma 2.3, it follows that

$$
\begin{aligned}
\prod_{l=1}^{k} \circ A_{l} & =\left(\prod_{l=1}^{k} \otimes A_{l}\right)(\alpha(k)) \\
& =\left(C^{*}\left(C^{*}\left(\prod_{l=1}^{k} \otimes A_{l}\right)^{-1} C\right)^{-1} C\right)(\alpha(k)) \\
& \geq C(\alpha(k))^{*}\left(C^{*}\left(\prod_{l=1}^{k} \otimes A_{l}\right)^{-1} C\right)(\alpha(k))^{-1} C(\alpha(k)) \\
& =C(\alpha(k))\left(C\left(\prod_{l=1}^{k} \otimes A_{l}\right)^{-1} C\right)(\alpha(k))^{-1} C(\alpha(k)),
\end{aligned}
$$

where $C(\alpha(k))=\sum_{t=1}^{k}\left(\left(\prod_{l \neq t} \circ A_{l}\right) \circ I\right)=N$, meanwhile

$$
\left(C\left(\prod_{l=1}^{k} \otimes A_{l}\right)^{-1} C\right)(\alpha(k))=\sum_{t=1}^{k}\left(A_{t}^{-1} \circ\left(\prod_{l \neq t} \circ A_{l}\right)\right)+2 \sum_{1 \leq t<s \leq k}\left(\left(\prod_{l \neq t, s} \circ A_{l}\right) \circ I\right) \in H(n),
$$

thus the inequality (3.4) follows by (3.3).

From Lemma 2.3 and the proof course as above, with a similar discussion as Theorem 3.1, we see the condition for the equation in (3.4) is determined by (3.5).

From above, the case discussed here is without 'positive definiteness', which is different from [1-10], and in form, the inequalities (3.1) and (3.4) are the reverses to each other; however, Theorems 3.1 and 3.3, and Example 3.2 indicate that their constraints are different, so (3.1) and (3.4) are not determined by each other any longer.

When $k=2$, we could obtain [8, Corollaries 2 and 3] from Theorems 3.1 and 3.3 immediately.

Corollary 3.4 Let $A \in H(n)$ be invertible, and $\alpha(k)$ be as in (2.7) and (2.8),

$$
C=\left(\sum_{t=1}^{k}\left(\prod_{l=1}^{t-1} \otimes A\right) \otimes I \otimes\left(\prod_{l=t+1}^{k} \otimes A\right)\right)
$$


(i) if $C$ is invertible and $\left(C^{-1}\left(\prod_{l=1}^{k} \otimes A\right) C^{-1}\right)\left(\alpha^{\prime}(k)\right)>0$, then $A^{-1} \circ\left(\prod_{l=1}^{k-1} \circ A\right)+(k-1)(A \circ I)^{k-2}$ is also invertible and the inequality (1.5) holds. Meanwhile, we have the equation in (1.5) if and only if

$$
\begin{aligned}
& k\left(C^{-1}\left(\prod_{l=1}^{k} \otimes A\right) C^{-1}\right)\left(\alpha^{\prime}(k), \alpha(k)\right)(A \circ I)^{k-1} \\
& \quad+\left(C^{-1}\left(\prod_{l=1}^{k} \otimes A\right) C^{-1}\right)\left(\alpha^{\prime}(k)\right) C\left(\alpha^{\prime}(k), \alpha(k)\right)=0 ;
\end{aligned}
$$

(ii) if $\left(\prod_{l=1}^{k} \otimes A^{-1}\right)\left(\alpha^{\prime}(k)\right)>0$, then $\prod_{l=1}^{k} \circ A \in H(n)$ is invertible and the inequality (2.17) holds. Meanwhile, the equation in (2.17) holds if and only if

$$
\begin{aligned}
& k\left(\prod_{l=1}^{k} \otimes A^{-1}\right)\left(\alpha^{\prime}(k), \alpha(k)\right)(A \circ I)^{k-1} \\
& \quad+\left(\prod_{l=1}^{k} \otimes A^{-1}\right)\left(\alpha^{\prime}(k)\right) C\left(\alpha^{\prime}(k), \alpha(k)\right)=0 .
\end{aligned}
$$

Proof In this case, $\sum_{t=1}^{k}\left(\left(\prod_{l=1}^{k-1} \circ A\right) \circ I\right)=k(A \circ I)^{k-1} \in H(n)$, by (3.3),

$$
\left(C^{*}\left(\prod_{l=1}^{k} \otimes A\right)^{-1} C\right)(\alpha(k))=k\left[A^{-1} \circ\left(\prod_{l=1}^{k-1} \circ A\right)+(k-1)(A \circ I)^{k-2}\right] \in H(n),
$$

then we could obtain the results by taking $A_{l}=A(l=1,2, \ldots, k)$ in Theorems 3.1 and 3.3.

Corollary 3.4 indicates that, without 'positive definiteness', not only the inequality (1.5) still holds under some constraints, but also its reverse inequality (2.17) still holds as well. Clearly their constraints are different.

Corollary 3.5 Let $A_{l} \in H(n)(l=1,2, \ldots, k)$ be invertible with all diagonal elements 1 and $\alpha(k)$ as in (2.7) and (2.8),

(i) if $\left(\prod_{l=1}^{k} \otimes A_{l}^{-1}\right)\left(\alpha^{\prime}(k)\right)>0$, then $\prod_{l=1}^{k} \circ A_{l}$ is invertible and

$$
k(k-1) I+\sum_{t=1}^{k}\left(A_{t}^{-1} \circ\left(\prod_{l \neq t} \circ A_{l}\right)\right) \geq k^{2}\left(\prod_{l=1}^{k} \circ A_{l}\right)^{-1}
$$

the equation in (3.8) holds if and only if

$$
k\left(\prod_{l=1}^{k} \otimes A_{l}^{-1}\right)\left(\alpha^{\prime}(k), \alpha(k)\right)+\left(\prod_{l=1}^{k} \otimes A_{l}^{-1}\right)\left(\alpha^{\prime}(k)\right) C\left(\alpha^{\prime}(k), \alpha(k)\right)=0 ;
$$

(ii) if $C$ is invertible and $\left(C^{-1}\left(\prod_{l=1}^{k} \otimes A_{l}\right) C^{-1}\right)\left(\alpha^{\prime}(k)\right)>0$, then $k(k-1) I+\sum_{t=1}^{k}\left(A_{t}^{-1} \circ\left(\prod_{l \neq t} \circ A_{l}\right)\right)$ is also invertible and

$$
\prod_{l=1}^{k} \circ A_{l} \geq k^{2}\left[k(k-1) I+\sum_{t=1}^{k}\left(A_{t}^{-1} \circ\left(\prod_{l \neq t} \circ A_{l}\right)\right)\right]^{-1} ;
$$


the equation in (3.10) holds if and only if

$$
\begin{aligned}
& k\left(C^{-1}\left(\prod_{l=1}^{k} \otimes A_{l}\right) C^{-1}\right)\left(\alpha^{\prime}(k), \alpha(k)\right) \\
& \quad+\left(C^{-1}\left(\prod_{l=1}^{k} \otimes A_{l}\right) C^{-1}\right)\left(\alpha^{\prime}(k)\right) C\left(\alpha^{\prime}(k), \alpha(k)\right)=0 .
\end{aligned}
$$

Proof By the assumption, $A_{l} \in H(n)(l=1,2, \ldots, k)$ is invertible with all diagonal elements 1 , so $N=\sum_{t=1}^{k}\left(\left(\prod_{l \neq t} \circ A_{l}\right) \circ I\right)=k I, 2 \sum_{1 \leq t<s \leq k}\left(\left(\prod_{l \neq t, s} \circ A_{l}\right) \circ I\right)=k(k-1) I$, then in view of (3.1), (3.2), (3.4), and (3.5) we have the conclusions.

For $A, B \in H^{+}(n)$, by (2.6), they satisfy the constraints demanded in Theorems 3.1 and 3.3 naturally. Hence similar to Theorem 2.6, by Lemma 2.4, we have the following.

Theorem 3.6 Let $A_{l} \in H^{+}(n)(l=1,2, \ldots, k)$ and $\alpha(k)$ be the one as in (2.7) and (2.8), then both of inequalities (3.1) and (3.4) hold, and the equation in (3.1) holds iff the one in (3.4) holds iff (3.2) holds iff (3.5) holds.

When $A=A_{l} \in H^{+}(n)(l=1,2, \ldots, k)$, by (1.8), one has

$$
\begin{gathered}
\left(A \circ I+I \circ \prod_{l=1}^{k-1} \circ A\right)\left(\prod_{l=1}^{k} \circ A\right)^{-1}\left(A \circ I+I \circ \prod_{l=1}^{k-1} \circ A\right) \\
\leq 2 I+\left(A \circ \prod_{l=1}^{k-1} \circ A^{-1}\right)+\left(A^{-1} \circ \prod_{l=1}^{k-1} \circ A\right) .
\end{gathered}
$$

Now in view of Theorem 2.5 and (2.17), we see the inequality (3.12) obtained from (1.8) is different from the one in (1.5). When $A=A_{l} \in H^{+}(n)(l=1,2, \ldots, k)$, by Theorem 3.6, we have the following.

Corollary 3.7 Let $A \in H^{+}(n)$ and $\alpha(k)$ be the one as in (2.7) and (2.8). Then both of inequalities (1.5) and (2.17) hold, and the equation in (1.5) holds iff the one in (2.17) holds iff (3.6) holds iff (3.7) holds.

By applying Theorem 3.6 and Corollaries 3.4, 3.5, we are led to the following conclusion.

Corollary 3.8 Let $A_{l} \in \mathrm{CH}^{+}(n)(l=1,2, \ldots, k), \alpha(k)$ be the one as in (2.7) and (2.8). Then both of inequalities (3.8) and (3.10) hold, moreover, the equation in (3.8) holds if and only if the one in (3.10) holds; thus (3.9) and (3.11) hold.

When $k=2$, the companion inequalities (1.1)-(1.4), (1.6), and (1.7), and their equation conditions are obtained.

The authors declare that they have no competing interests. 


\section{Author details}

${ }^{1}$ School of Mathematics, Putian University, Putian, 351100, P.R. China. ${ }^{2}$ School of Mathematics and Computer Science, Fujian Normal University, Fuzhou, 350007, P.R. China. ${ }^{3}$ School of Mathematics and Statistics, Minnan Normal University, Zhangzhou, 363000, P.R. China.

\section{Acknowledgements}

The work is supported by the National Natural Science Foundation of China (No. 61373140), the Natural Science Foundation of Fujian Province (No. 2013J00102), the middle-aged research item in Education Committee of Fujian Province (No. JA14277), the key item of Hercynian building for the colleges and universities service in Fujian Province (2008HX03) and the teaching reformation project of Putian University (JG201415).

Received: 15 May 2014 Accepted: 18 November 2014 Published: 02 Dec 2014

\section{References}

1. Styan, GPH: Hadamard products and multivariate statistical analysis. Linear Algebra Appl. 6, 217-240 (1973)

2. Ando, T: Concavity of certain maps on positive definite matrices and applications for Hadamard products. Linear Algebra Appl. 26, 203-241 (1979)

3. Zhang, F: Schur complements and matrix inequalities in the Loewner ordering. Linear Algebra Appl. 321, 399-410 (2000)

4. Visick, G: A quantitative version of the observation that the Hadamard product is a principal submatrix of the Kronecker product. Linear Algebra Appl. 304, 45-68 (2000)

5. Liu, S, Trenkler, G: Hadamard, Khatri-Rao, Kronecker and other matrix products. Int. J. Inf. Syst. Sci. 4, 160-177 (2008)

6. Al Zhour, ZAA, Kilicman, A: Extension and generalization inequalities involving the Khatri-Rao product of several positive matrices. J. Inequal. Appl. 2006, Article ID 80878 (2006)

7. Liu, S: Inequalities involving Hadamard products of positive semidefinite matrices. J. Math. Anal. Appl. 243, 458-463 (2000)

8. Yang, ZP, Liu, S, Trenkler, G: Further inequalities involving the Khatri-Rao product. Linear Algebra Appl. 430, 2696-2704 (2009)

9. Yang, ZP, LV, HB, Feng, XX: Generalization of reverse Styan matrix inequalities. J. Xiamen Univ., Nat. Sci. 47(1), 7-11 (2008)

10. Mond, B, Pecaric, J: On inequalities involving Hadamard product of matrices. Electron. J. Linear Algebra 6, 56-61 (2000)

11. Yang, ZP, Feng, XX: The equivalent form of a matrix inequality and its application. J. Appl. Math. Comput. 20(1/2), 421-431 (2006)

12. Liu, S, Polasck, W, Neudecker, H: Equality conditions for matrix Kantorovich-type inequalities. J. Math. Anal. Appl. 212 , 517-528 (1997)

13. Markham, TL, Smith, RL, Bork, P: A Schur complement inequality for certain P-matrices. Linear Algebra Appl. 281, 33-41 (1998)

14. Horn, RA, Johnson, CR: Topics in Matrix Analysis. Cambridge University Press, New York (1991)

15. Bernstein, DS: Matrix Mathematics: Theory, Facts, and Formulas, 2nd edn. Princeton University Press, Princeton (2009)

16. Cao, CG, Zhang, X, Yang, ZP: Some inequalities for the Khatri-Rao product of matrices. Electron. J. Linear Algebra 9 276-281 (2002)

17. Feng, BQ: On the explicit selection matrix of relating the tensor products and Hadamard products of matrices. Far East J. Math. Sci. 39(2), 209-219 (2010)

18. Wang, BY, Zhang, F: Schur complements and matrix inequalities of Hadamard products. Linear Multilinear Algebra 43, 315-326 (1997)

19. Zhang, F: The Schur Complements and Its Applications. Springer, New York (2005)

10.1186/1029-242X-2014-479

Cite this article as: Chen et al.: The further generalization on the inequalities for Hadamard products of any number of invertible Hermitian matrices. Journal of Inequalities and Applications 2014, 2014:479

\section{Submit your manuscript to a SpringerOpen ${ }^{\ominus}$ journal and benefit from:}

- Convenient online submission

- Rigorous peer review

- Immediate publication on acceptance

Open access: articles freely available online

- High visibility within the field

- Retaining the copyright to your article 